PROCEEDINGS OF THE

AMERICAN MATHEMATICAL SOCIETY

Volume 135, Number 11, November 2007, Pages 3507-3514

S 0002-9939(07)08883-1

Article electronically published on July 27, 2007

\title{
AN ARITHMETIC FORMULA FOR THE PARTITION FUNCTION
}

\author{
KATHRIN BRINGMANN AND KEN ONO \\ (Communicated by Wen-Ching Winnie Li)
}

\section{Introduction AND STATEMEnt of REsults}

A partition of a non-negative integer $n$ is a non-increasing sequence of positive integers whose sum is $n$. As usual, let $p(n)$ denote the number of partitions of $n$. The generating function for $p(n)$ is given by the infinite product

$$
\sum_{n=0}^{\infty} p(n) q^{n}=\prod_{n=1}^{\infty} \frac{1}{1-q^{n}}
$$

Although there is a vast literature on the properties of $p(n)$, typically motivated by work of Ramanujan, some of the simplest questions remain open. For example, little is known about $p(n)$ modulo 2 and 3. Most results concerning the congruence properties of $p(n)$ have been proved using properties of (1.1). Theorems typically depend on $q$-series identities, the theory of modular equations, or the theory of Hecke operators acting on $p$-adic modular forms. Here we propose a new approach, one based on the properties of algebraic numbers associated to CM points.

Before we discuss the partition function, we begin by considering the $q$-series

$$
\sum_{n=-1}^{\infty} a(n) q^{n}:=q^{-1} \prod_{n=1}^{\infty} \frac{\left(1-q^{n}\right)}{\left(1+q^{n}\right)\left(1-q^{4 n}\right)^{6}}=q^{-1}-2+8 q^{3}-12 q^{4}+39 q^{7}-\cdots
$$

It turns out that the following congruences hold for the coefficients $a(n)$.

Theorem 1.1. For every non-negative integer $n$, we have

$$
\begin{aligned}
& a(8 n+3) \equiv 0 \quad(\bmod 8), \\
& a(3 n+1) \equiv 0 \quad(\bmod 3) .
\end{aligned}
$$

Furthermore, if $-n \equiv 0,1(\bmod 4)$ is negative, then

$$
a(n) \equiv\left\{\begin{array}{lll}
8 H(-n) & (\bmod 16) & \text { if } n \equiv 3 \quad(\bmod 8) \\
4 H(-n) & (\bmod 5) & \text { if }\left(\frac{n}{5}\right)=-1,
\end{array}\right.
$$

where $H(-n)$ is the Hurwitz-Kronecker class number for $-n$.

Received by the editors September 17, 2005 and, in revised form, August 23, 2006.

2000 Mathematics Subject Classification. Primary 11P82.

The authors thank the National Science Foundation for their generous support.

The second author is grateful for the support of a Packard, a Romnes, and a Guggenheim Fellowship. 
The congruences modulo 8 and 3 are easy to prove using $q$-series manipulations, or the theory of Hecke operators. Instead we give an "arithmetic" proof, uniformly describing all four congruences. Such arguments, when they apply, arithmetically explain the congruence properties of integers like $a(n)$ modulo small powers of small primes.

Although we defer the proof of Theorem 1.1 to Section 2 here we illustrate the simple idea underlying the proof by explaining the congruence

$$
a(35)=69168 \equiv 0 \quad(\bmod 16) .
$$

The class number $H(-35)$ denotes the number of classes of positive definite integral binary quadratic forms with discriminant -35 under the action of $\mathrm{PSL}_{2}(\mathbb{Z})$. It turns out that $H(-35)=2$, and one may represent these two classes of quadratic forms by

$$
Q_{1}(x, y):=x^{2}+x y+9 y^{2} \quad \text { and } \quad Q_{2}(x, y):=3 x^{2}+x y+3 y^{2} .
$$

If $\tau_{Q_{1}}$ and $\tau_{Q_{2}}$ are the unique points in the upper half of the complex plane which are roots of $Q_{1}(x, 1)=0$ and $Q_{2}(x, 1)=0$, respectively, and if $j(z)$ is the usual modular $j$-function

$$
j(z)=q^{-1}+744+196884 q+21493760 q^{2}+\cdots
$$

$\left(q:=e^{2 \pi i z}\right.$ throughout), then it is a classical fact that

$j\left(\tau_{Q_{1}}\right)=-58982400-26378240 \sqrt{5} \quad$ and $\quad j\left(\tau_{Q_{2}}\right)=-58982400+26378240 \sqrt{5}$.

These numbers satisy the congruence

$$
j\left(\tau_{Q_{i}}\right)-744 \equiv 8 \quad(\bmod 16) .
$$

Thanks to recent work of Zagier 4, it is not difficult to show that

$$
\left.a(35) \equiv-\left(j\left(\tau_{Q_{1}}\right)-744\right)\right)-\left(j\left(\tau_{Q_{2}}\right)-744\right)=117966288 \quad(\bmod 240) .
$$

Therefore, congruence (1.3) follows immediately from (1.4) and (1.5).

The proof of Theorem 1.1 depends on the fact that (1.4) and (1.5) hold in greater generality. In view of this arithmetic proof for the $a(n)$ congruences, it is natural to ask whether such an approach can be made to apply to the partition function $p(n)$. Success of such a strategy requires analogs of both (1.4) and (1.5). Here we take the first step and obtain an analog of (1.5). In other words, we show that $p(n)$ is a "trace" of CM values of a fixed modular invariant.

To state this result, we first fix notation. Let $d \equiv 0,3(\bmod 4)$ be a positive integer, and let $\mathcal{Q}_{d}^{(p)}$ be the set of positive definite integral binary quadratic forms (note: including imprimitive forms, if there are any)

$$
Q(x, y)=[a, b, c]=a x^{2}+b x y+c y^{2}
$$

of discriminant $-d=b^{2}-4 a c$, with the additional property that $6 \mid a$. The congruence subgroup $\Gamma_{0}(6)$ acts on $\mathcal{Q}_{d}^{(p)}$. Specifically, if $M=\left(\begin{array}{cc}\alpha & \beta \\ \gamma & \delta\end{array}\right) \in \Gamma_{0}(6)$, then this action is defined by

$$
Q \circ M:=[a, b, c](\alpha x+\beta y, \gamma x+\delta y):=\left[a^{\prime}, b^{\prime}, c^{\prime}\right] .
$$

Since $6 \mid a^{\prime}$, it follows that this action is well defined.

For each $Q$, let $\tau_{Q}$ be the unique complex number in $\mathbb{H}$, the upper half of the complex plane, which is a root of $Q(x, 1)=0$, and let $\Gamma_{\tau_{Q}}$ be its isotropy subgroup 
in $\Gamma_{0}(6)$. If $f(z): \mathbb{H} \rightarrow \mathbb{C}$ is a $\Gamma_{0}(6)$-invariant function, then for a positive integer $n$ we define the discriminant $-24 n+1$ twisted trace of $f(z)$, say $\operatorname{Tr}^{(p)}(f ; n)$, by

$$
\operatorname{Tr}^{(p)}(f ; n):=\sum_{Q \in \mathcal{Q}_{24 n-1}^{(p)} / \Gamma_{0}(6)} \frac{\chi_{12}(Q) f\left(\tau_{Q}\right)}{\# \Gamma_{\tau_{Q}}},
$$

where $\chi_{12}(Q)$ is defined by

$$
\chi_{12}([a, b, c]):=\left(\frac{12}{b}\right) .
$$

Remark. It is simple to check that $b \equiv b^{\prime}(\bmod 12)$ in (1.6). Therefore, formula (1.7) is well defined.

The partition numbers $p(n)$ are traces of a Poincaré series $P(z)$ which is a weakly holomorphic Maass form. To define this series, let $I_{s}(x)$ denote the usual $I$-Bessel function, the so-called modified Bessel function of the first kind, and let

$$
\Gamma_{\infty}:=\left\{ \pm\left(\begin{array}{cc}
1 & n \\
0 & 1
\end{array}\right): n \in \mathbb{Z}\right\}
$$

denote the translations within $\mathrm{SL}_{2}(\mathbb{Z})$. This Poincaré series is defined by

$$
P(z):=4 \pi \sum_{A \in \Gamma_{\infty} \backslash \Gamma_{0}(6)} \operatorname{Im}(A z)^{\frac{1}{2}} I_{\frac{3}{2}}(2 \pi \operatorname{Im}(A z)) e(-\operatorname{Re}(A z)),
$$

where for $x \in \mathbb{R}$ we let $e(x):=e^{2 \pi i x}$.

Theorem 1.2. If $n$ is a positive integer, then

In particular, we have that

$$
p(n)=\frac{\operatorname{Tr}^{(p)}(P ; n)}{24 n-1} .
$$

$$
p(n) \equiv-\operatorname{Tr}^{(p)}(P ; n) \quad(\bmod 24) .
$$

Theorem 1.2 is the partition function analog of (1.5). In both cases we have power series coefficients expressed as sums of a modular invariant over equivalence classes of CM points. In (1.5) we have traces of $j(z)-744$ modulo 240, and in Theorem 1.2 we have an exact identity describing $p(n)$ as traces of $P(z)$. It turns out that these two facts have even more in common. If $s>1$ and

$$
F_{s}(z):=-12+\pi \sum_{A \in \Gamma_{\infty} \backslash S L_{2}(\mathbb{Z})} \operatorname{Im}(A z)^{\frac{1}{2}} I_{s-\frac{1}{2}}(2 \pi \operatorname{Im}(A z)) e(-\operatorname{Re}(A z))
$$

(compare with (1.8)), then Niebur [2] showed that the analytic continuation of $F_{s}(z)$, as $s \rightarrow 1$, is $\frac{1}{2}(j(z)-744)$. Consequently, in both cases the coefficients are traces of a Poincaré series of similar type.

Questions. In view of these results and analogies, it is natural to raise the following questions:

(1) Is there a simple description of $P(z)$ in terms of

$$
E_{2}^{*}(z)=-\frac{3}{\pi \operatorname{Im}(z)}+1-24 \sum_{n=1}^{\infty} \sigma_{1}(n) q^{n}
$$

and canonical modular forms such as Eisenstein series and quotients of Dedekind-eta functions? 
(2) Are there suitable algebraic normalizations of the values of $P(z)$ at $\mathrm{CM}$ points?

(3) If so, then are there analogs of congruences such as (1.4) for these normalizations?

Remark. A positive solution to question (1) is presumably the most direct approach to question (2).

In Section 2 we recall classical congruence properties for values of the $j$-function, and we recall Zagier's work on traces of singular moduli. Then in Section 3 we prove Theorem 1.1. Theorem 1.2 is simply an arithmetic reformulation of Rademacher's exact formula for $p(n)$. In Section 4 we recall a useful version of this formula which describes $p(n)$ as an infinite sum involving $I$-Bessel functions and Salié-type sums. We then use the classical fact that such Salié sums may be rewritten as Poincarétype series over orbits of CM points to prove Theorem 1.2. In Section 5 we conclude by describing the Poincaré series $P(z)$ as a Maass form.

\section{Singular moduli ANd the Proof of Theorem 1.1}

Here we prove Theorem 1.1. The proof follows from the generalizations of (1.4) and (1.5). We begin by recalling these generalizations.

As before, let

$$
j(z)=q^{-1}+744+196884 q+21493760 q^{2}+\cdots
$$

be the usual modular $j$-function. Its values at CM points in the upper half of the complex plane are known as singular moduli, and they are algebraic integers.

The facts we require about singular moduli are easily described in the language of quadratic forms. To make this precise, let $d \equiv 0,3(\bmod 4)$ be a positive integer, and let $\mathcal{Q}_{d}$ be the set of positive definite integral binary quadratic forms (note: including imprimitive forms, if there are any)

$$
Q(x, y)=[a, b, c]=a x^{2}+b x y+c y^{2}
$$

with discriminant $-d=b^{2}-4 a c$. For each $Q$, let $\tau_{Q}$ be the unique complex number in the upper half-plane which is a root of $Q(x, 1)=0$.

It is a classical fact that the singular modulus $j\left(\tau_{Q}\right)$ is an algebraic integer which depends only on the equivalence class of $Q$ under the action of $\Gamma:=\operatorname{PSL}_{2}(\mathbb{Z})$. Following Zagier, we use these singular moduli to define certain traces which turn out to be integers closely related to the integers $a(n)$ in Theorem 1.1. If $\omega_{Q} \in$ $\{1,2,3\}$ is given by

$$
\omega_{Q}:= \begin{cases}2 & \text { if } Q \sim_{\Gamma}[a, 0, a], \\ 3 & \text { if } Q \sim_{\Gamma}[a, a, a], \\ 1 & \text { otherwise }\end{cases}
$$

then define the trace $\operatorname{Tr}(j-744 ; d)$ by

$$
\operatorname{Tr}(j-744 ; d):=\sum_{Q \in \mathcal{Q}_{d} / \Gamma} \frac{j\left(\tau_{Q}\right)-744}{\omega_{Q}} .
$$

Zagier proved (see Theorem 1 of 4]) the following theorem concerning the generating function of these traces. 
Theorem 2.1. If integers $b(n)$ are defined by

$$
\sum_{n=-1}^{\infty} b(n) q^{n}:=\left(\sum_{n=-1}^{\infty} a(n) q^{n}\right) \cdot\left(1+240 \sum_{n=1}^{\infty} \sum_{d \mid n} d^{3} q^{4 n}\right)=q^{-1}-2+248 q^{3}-\cdots
$$

then for every positive integer $d \equiv 0,3(\bmod 4)$ we have

$$
\operatorname{Tr}(j-744 ; d)=-b(d) .
$$

Example. To illustrate Theorem 2.1, consider the case where $d=3$. We have $H(-3)=1 / 3$, and the quadratic form $Q=x^{2}+x y+y^{2}$ can be chosen to represent $\mathcal{Q}_{3} / \Gamma$. Therefore, it follows that

$$
\operatorname{Tr}(j-744 ; 3)=\frac{j\left(\frac{-1+\sqrt{-3}}{2}\right)-744}{3}=-248 .
$$

The fact that $b(3)=248$ confirms the $d=3$ case of Theorem 2.1.

It turns out that singular moduli satisfy some delightfully simple congruences modulo small powers of small primes. We require the following congruences proven by Gross and Zagier in [1].

Theorem 2.2. If $d \equiv 0,3(\bmod 4)$ is a positive integer and $Q \in \mathcal{Q}_{d}$, then

$$
\begin{aligned}
d \equiv 3 \quad(\bmod 8) & \Longrightarrow j\left(\tau_{Q}\right) \equiv 0 \quad\left(\bmod 2^{15}\right) \\
d \equiv 1 \quad(\bmod 3) & \Longrightarrow j\left(\tau_{Q}\right) \equiv 1728 \quad\left(\bmod 3^{6}\right), \\
d \equiv 2,3 \quad(\bmod 5) & \Longrightarrow j\left(\tau_{Q}\right) \equiv 0 \quad\left(\bmod 5^{3}\right) .
\end{aligned}
$$

\section{Proof of Theorem 1.1}

Here we use the facts given in Section 2 to prove Theorem 1.1. By construction, the integers $b(n)$ defined in the statement of Theorem 2.1 satisfy the congruence

$$
a(n) \equiv b(n) \quad(\bmod 240)
$$

for all $n$. Therefore, it suffices to prove the claimed congruences for the integers $b(n)$.

Since we have that

$$
H(-d)=\sum_{Q \in \mathcal{Q}_{d} / \Gamma} \frac{1}{\omega_{Q}}
$$

equation (2.1) and the congruences in Theorem 2.2 imply that

$$
\begin{aligned}
& d \equiv 3 \quad(\bmod 8) \quad \Longrightarrow \quad \operatorname{Tr}(j-744 ; d) \equiv 8 H(-d) \quad(\bmod 16), \\
& d \equiv 1 \quad(\bmod 3) \Longrightarrow \operatorname{Tr}(j-744 ; d) \equiv 0 \quad(\bmod 3), \\
& d \equiv 2,3 \quad(\bmod 5) \quad \Longrightarrow \quad \operatorname{Tr}(j-744 ; d) \equiv H(-d) \quad(\bmod 5) \text {. }
\end{aligned}
$$

Theorem 1.1 now follows by applying Theorem 2.1 . 


\section{Rademacher's sums ANd the Proof of Theorem 1.2}

Theorem 1.2 is an arithmetic reformulation of Rademacher's exact formula for $p(n)$. Here we recall one version of his famous result (see pages 273 and 282 of [3]).

Theorem 4.1. If $n$ is a positive integer, then

$$
p(n)=2 \pi(24 n-1)^{-\frac{3}{4}} \sum_{k=1}^{\infty} \frac{A_{k}(n)}{k} I_{\frac{3}{2}}\left(\frac{\pi \sqrt{24 n-1}}{6 k}\right),
$$

where

$$
A_{k}(n):=\frac{1}{2} \sqrt{\frac{k}{12}} \sum_{\substack{x \\ x^{2} \equiv-24 n+1 \quad(\bmod 24 k)}}(-1)^{\left\{\frac{x}{6}\right\}} \cdot e\left(\frac{x}{12 k}\right) .
$$

In the sum, $x$ runs through the residue classes modulo $24 k$, and $\{\alpha\}$ denotes the integer nearest to $\alpha$.

Remark. Note that $\left\{\frac{x}{6}\right\}$ is well defined for those $x$ appearing in the definition of $A_{k}(n)$.

The sums $A_{k}(n)$ are Salié type sums, and they are easily described in terms of orbits of CM points under the action of $\Gamma_{0}(6)$.

Proposition 4.2. If $k$ and $n$ are positive integers, then we have

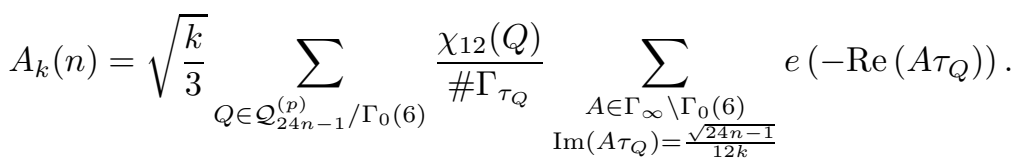

Proof. For every integral binary quadratic form

$$
Q(x, y):=6 k x^{2}+b x y+c y^{2}
$$

of discriminant $-24 n+1$, we have that

$$
\tau_{Q}=\frac{-b+i \sqrt{24 n-1}}{12 k} .
$$

Obviously, the coefficient $b$ of $Q$ solves the congruence

$$
b^{2} \equiv-24 n+1 \quad(\bmod 24 k) .
$$

Conversely, every solution of (4.2) corresponds to a quadratic form with an associated CM point as above. Therefore there is a one-to-one correspondence between the solutions of

$$
b^{2}-24 k c=-24 n+1 \quad(k, b, c \in \mathbb{Z} \text { with } k, c>0)
$$

and the points of the orbits

$$
\bigcup_{\substack{(p) \\ Q \mathcal{Q}_{24 n-1}^{(p)} / \Gamma_{0}(6)}}\left\{A \tau_{Q}: A \in \Gamma_{0}(6) / \Gamma_{\tau_{Q}}\right\} .
$$

The group $\Gamma_{\infty}$ preserves the imaginary part of such a CM point $\tau_{Q}$, and preserves (4.2). However, it does not preserve the middle coefficient $b$ of the corresponding quadratic forms modulo $24 k$. It identifies (but does not distinguish) the congruence classes $b, b+12 k(\bmod 24 k)$ occuring in the definition of $A_{k}(n)$. Since $\chi_{12}(Q)=$ $(-1)^{\left\{\frac{b}{6}\right\}}$ (see (124.1) on page 283 of [3] ) is fixed under the action of $\Gamma_{0}(6)$, which includes $\Gamma_{\infty}$, the corresponding summands for such pairs of congruence classes are 
equal. The proposition now follows easily since $\Gamma_{\tau_{Q}}$ contains the negative identity matrix.

Proof of Theorem 1.2. Theorem 4.1 and Proposition 4.2 give

$$
\begin{aligned}
p(n)=\frac{2 \pi}{\sqrt{3}}(24 n-1)^{-\frac{3}{4}} & \sum_{\substack{Q \in \mathcal{Q}_{24 n-1}^{(p)} / \Gamma_{0}(6)\\
}} \frac{\chi_{12}(Q)}{\# \Gamma_{\tau_{Q}}} \\
& \sum_{k=1}^{\infty} \frac{1}{\sqrt{k}} \sum_{\substack{A \in \Gamma_{\infty} / \Gamma_{0}(6) \\
\operatorname{Im}\left(A \tau_{Q}\right)=\frac{\sqrt{24 n-1}}{12 k}}} I_{\frac{3}{2}}\left(2 \pi \operatorname{Im}\left(A \tau_{Q}\right)\right) \cdot e\left(-\operatorname{Re}\left(A \tau_{Q}\right)\right) .
\end{aligned}
$$

The definition of $P(z)$, combined with the obvious change of variable relating $1 / \sqrt{k}$ to $\operatorname{Im}\left(A \tau_{Q}\right)^{\frac{1}{2}}$, gives

$$
\begin{aligned}
p(n)= & \frac{4 \pi}{24 n-1} \sum_{Q \in \mathcal{Q}_{24 n-1}^{(p)} / \Gamma_{0}(6)} \frac{\chi_{12}(Q)}{\# \Gamma_{\tau_{Q}}} \\
& \times \sum_{A \in \Gamma_{\infty} / \Gamma_{0}(6)} \operatorname{Im}\left(A \tau_{Q}\right)^{\frac{1}{2}} I_{\frac{3}{2}}\left(2 \pi \operatorname{Im}\left(A \tau_{Q}\right)\right) e\left(-\operatorname{Re}\left(A \tau_{Q}\right)\right) \\
= & (24 n-1)^{-1} \operatorname{Tr}^{(p)}(P ; n) .
\end{aligned}
$$

\section{The Poincaré series $P(z)$}

Here we discuss the properties of the Poincaré series $P(z)$. By definition, we have

$$
P(z):=4 \pi \sum_{A \in \Gamma_{\infty} \backslash \Gamma_{0}(6)} \operatorname{Im}(A z)^{\frac{1}{2}} I_{\frac{3}{2}}(2 \pi \operatorname{Im}(A z)) e(-\operatorname{Re}(A z)) .
$$

Since we have

$$
y^{1 / 2} I_{\frac{3}{2}}(y)=O\left(y^{2}\right) \quad(y \rightarrow 0),
$$

it follows that the defining series for $P(z)$ is absolutely uniformly convergent. Moreover, its definition implies that if $M \in \Gamma_{0}(6)$, then

$$
P(M z)=P(z)
$$

for all $z=x+i y \in \mathbb{H}$. Therefore, $P(z)$ is a $\Gamma_{0}(6)$-invariant function on $\mathbb{H}$.

It is a weight 0 weakly holomorphic Maass form for $\Gamma_{0}(6)$. Using the properties of $I_{\frac{3}{2}}(x)$, the function

$$
f(z):=\pi y^{\frac{1}{2}} I_{\frac{3}{2}}(2 \pi y) e(-x)
$$

is easily shown to satisfy

$$
\Delta(f(z))=-2 f(z)
$$

where

$$
\Delta:=-y^{2}\left(\frac{\partial^{2}}{\partial x^{2}}+\frac{\partial^{2}}{\partial y^{2}}\right)
$$

is the usual weight 0 hyperbolic Laplacian. Thus, it follows that

$$
\Delta(P(z))=-2 P(z) .
$$




\section{REFERENCES}

[1] B. Gross and D. Zagier, On singular moduli, J. Reine Angew. Math. 355 (1985), 191-220. MR772491 (86j:11041)

[2] D. Niebur, A class of nonanalytic automorphic functions, Nagoya Math. J. 52 (1973), 133145. MR0337788 (49:2557)

[3] H. Rademacher, Topics in analytic number theory, Die Grundlehren der mathematischen Wissenschaften, Band 169. Springer Verlag New York- Heidelberg, 1973. MR0364103 (51:358)

[4] D. Zagier, Traces of singular moduli, Motives, polylogarithms and Hodge theory, Part I (Irvine, CA, 1998) (2002), Int. Press Lect. Ser., 3, I, Int. Press, Somerville, MA, 211-244. MR 1977587 (2004h:11037)

Department of Mathematics, University of Wisconsin, Madison, Wisconsin 53706

E-mail address: bringman@math.wisc.edu

Department of Mathematics, University of Wisconsin, Madison, Wisconsin 53706

E-mail address: ono@math.wisc.edu 